\title{
Public und Global Health in Deutschland stärken - Eine Einordnung der Stellungnahme „Public Health in Deutschland“ der wissenschaftlichen Akademien vom 16.6.2015
}

\section{Strengthening Public and Global Health in Germany - an Appraisal of the Statement "Public Health in Germany" by the German Scientific Academies from June 16th 2015}

Autoren

Institute
A. Gerhardus ${ }^{1,2}$, B. Blättner ${ }^{3}$, G. Bolte ${ }^{2,4}$, M.-L. Dierks ${ }^{5}$, C. Dockweiler ${ }^{6}$, G. Faller ${ }^{7}$, B. Gusy ${ }^{8}$ (Vorstand der Deutschen Gesellschaft für Public Health)

Die Institutsangaben sind am Ende des Beitrags gelistet
Schlüsselwörter

- Public Health

- Global Health

- Deutschland

- Forschungsstrukturen

- Ausbildung

Key words

public health

- global health

- Germany

- research structures

- education

\section{Bibliografie}

DOI http://dx.doi.org/

10.1055/s-0035-1559692

Gesundheitswesen 2015;

77: 596-599

(c) Georg Thieme Verlag KC

Stuttgart · New York

ISSN 0941-3790

Korrespondenzadresse

Prof. Dr. med. Ansgar

Gerhardus, MA, MPH

1. Vorsitzender

Deutsche Gesellschaft für

Public Health (DGPH)

Abteilung 1: Versorgungs-

forschung

Institut für Public Health und

Pflegeforschung

Fachbereich 11

Universität Bremen

Grazer Straße 4

28359 Bremen

ansgar.gerhardus@uni-

bremen.de

\section{Zusammenfassung}

Im Juni 2015 haben die wissenschaftlichen Akademien Leopoldina und acatech und die Union der Deutschen Akademien der Wissenschaften eine Stellungnahme zu Public und Global Health in Deutschland veröffentlicht. Die Stellungnahme enthält eine Reihe von wertvollen, aber allgemein gehaltenen und weitgehend bekannten Empfehlungen. Die wenigen konkreten Empfehlungen sehen eine Zentralisierung der Forschung und eine Abwertung der Rolle der Universitäten vor. Gegenüber anderen Bereichen soll Public Health Genomics weiter ausgebaut werden. Diese Empfehlungen werden durch keine empirischen oder theoretischen Analysen gestützt und laufen Gefahr, die erfolgreichen Prozesse der letzten Jahre umzukehren. Um Public und Global Health in Deutschland wirksam zu stärken sollten stattdessen die vorhandenen Institutionen besser unterstützt und die Forschungsförderung ausgebaut werden.

Am 16. Juni 2015 haben die wissenschaftlichen Akademien Leopoldina und acatech und die Union der Deutschen Akademien der Wissenschaften eine Stellungnahme zu Public und Global Health in Deutschland veröffentlicht [1]. Neben der Forderung nach einer materiellen und immateriellen Aufwertung von Public und Global Health in Praxis, Forschung und Lehre in Deutschland werden dort weitreichende strukturelle und inhaltliche Vorschläge unterbreitet - allerdings ohne erkennbare theoretische oder empirische Fundierung. Eine Umsetzung der Empfehlungen birgt die Gefahr, die positiven Entwicklungen der jüngsten Zeit wieder umzukehren.

\section{Abstract \\ $\nabla$}

In June 2015 the scientific academies "Leopoldina" and "acatech" and the union of the German academies of sciences and humanities published a statement on public and global health in Germany. The statement contains many valuable albeit generic and well-known recommendations. The few specific recommendations focus on centralizing research while weakening the role of universities and on the relative increase of research on public health genomics. These recommendations are not based on empirical or theoretical analyses and risk subverting successful developments of the recent past. To actually strengthen public and global health in Germany the existing institutions need to be backed up while designated funding of public and global health needs to be extended.

\section{Worum geht es in der Stellungnahme der Akademien? \\ $\nabla$}

Wissenschaft, Lehre und Praxis von Public und Global Health in Deutschland wird im Vergleich zu anderen Ländern ein Nachholbedarf attestiert. Dieser wird auf ungünstige strukturelle Voraussetzungen von Public Health und auf eine „Zersplitterung“ von Forschung, Lehre und Praxis zurückgeführt. Entsprechend sehen die Empfehlungen unterschiedlich ausgeprägte Maßnahmen der Zentralisierung bzw. Koordinierung vor. Die Universitäten, die bisher das Rückgrat der Forschung bilden, sollen zukünftig auf die Rolle von „wichtigen Partnern“ reduziert werden, deren Zweck es ist ,....Lehre und Ausbildung im Bereich Public Health zu stärken.“ 


\section{Was sind die Stärken der Stellungnahme? P}

Die Akademien verfügen über nachhaltigen Einfluss in der Wissenschaftspolitik, auch wenn ihre fachliche Expertise nicht primär im Bereich von Public und Global Health liegt. Es ist ihnen gelungen, einige in Public Health oder Global Health hervorragend ausgewiesene, nationale und internationale Wissenschaftlerinnen und Wissenschaftler einzubeziehen. Der Forderung nach einer Stärkung von Public und Global Health könnte dadurch bei politischen Entscheidungsträgern mehr Gewicht verschafft werden.

In der Stellungnahme werden nahezu alle Themen angerissen, die für Public und Global Health von Relevanz sind. Hervorgehoben wird, dass die Zusammenarbeit vieler Disziplinen und Akteure, eine solide Finanzierung und eine bessere Verzahnung zwischen Wissenschaft, Politik und Praxis notwendig sind. Diese Gedanken sind nicht neu, werden aber durch die Aussagen der wissenschaftspolitisch einflussreichen Institutionen bestätigt und gewinnen dadurch an Nachdruck.

\section{Was sind die Schwächen der Stellungnahme? \\ $\nabla$}

Wer eine systematische Analyse erwartet, wird enttäuscht: Eine theoretisch oder empirisch fundierte Argumentation ist nicht erkennbar. Dies überrascht, da im Entstehungsprozess mehrere Workshops mit eingeladenen Expertinnen und Experten stattgefunden haben. Diese scheinen aber wenig Einfluss auf das finale Papier genommen zu haben. Bspw. bleibt die Argumentation in der Box 4, Diskussionsthemen des Workshops „Lebensbedingungen und Kausalität“ (S. 28 f) und der Box 5, Diskussionsthemen des Workshops „Prävention und Gesundheitsförderung“ (S. 31), oberflächlich und ist in keinem Punkt innovativ. Es ist kaum vorstellbar, dass damit die Diskussionsthemen in den Workshops adäquat abgebildet sind.

Ein wirklicher Vergleich von Public-Health-Entwicklungen in Deutschland mit den Voraussetzungen und Strukturen der Länder, die in dem Text als Referenz geführt werden, findet nicht statt. Der Abschnitt unter der Überschrift „Internationales Benchmarking“ (S. 59 ff) enthält keine spezifischen Informationen, sondern mündet in der Empfehlung „... sich nicht zu sehr auf internationale Vergleiche oder die Vergangenheit zu fixieren, ..."(S. 59). Der einzige Versuch eines Vergleichs, die bibliometrische Analyse, bietet teilweise verzerrte Ergebnisse und geht auf den positiven Befund einer im globalen Vergleich überdurchschnittlichen Steigerung der Publikationszahlen in Deutschland in der letzten Dekade nicht ein (s.u.).

Die Darstellung von Forschung und Lehre in Public Health sowie die problematisierte Zusammenarbeit mit der Praxis beruhen auf teilweise profunden Missverständnissen und einer begrenzten Kenntnis der laufenden Aktivitäten (s.u.).

\section{Gegenüberstellung von zentralen Punkten der Stellungnahme und den vorhandenen Fakten $\nabla$}

\section{Forderung nach Zentralisierung von Public und Global} Health in Deutschland

Der Wunsch nach Zentralisierung wird mit dem Hinweis auf eine angebliche Zersplitterung der akademischen Landschaft auf dem Gebiet von Public und Global Health begründet. Wie sehen die Fakten dazu aus?
Aktuell wird Forschung zu Public und Global Health in Deutschland primär an Universitäten und Hochschulen für angewandte Wissenschaften unter wettbewerblichen Bedingungen geleistet. Dazu kommt im Wesentlichen die Forschung durch öffentlich finanzierte Forschungseinrichtungen (z.B. der Helmholtz- oder der Leibniz-Gemeinschaft) und von Behörden des Bundes und der Länder mit partiellem Forschungsauftrag (z.B. Robert Koch-Institut, Landesgesundheitsämter). Die Hochschulen haben jeweils individuelle Profile entwickelt und arbeiten mit vielfältigen Kooperationspartnern zusammen. Dazu einige Beispiele:

- Das Institut für Epidemiologie, Sozialmedizin und Gesundheitssystemforschung der Medizinischen Hochschule Hannover arbeitet eng mit den einzelnen klinischen Disziplinen zusammen und hat eine „Patientenuniversität“ etabliert;

- die School of Public Health der Universität Bielefeld kooperiert mit der dortigen soziologischen Fakultät und verfolgt einen sozialwissenschaftlichen Schwerpunkt in Public Health;

- der Fachbereich Human- und Gesundheitswissenschaften der Universität Bremen kooperiert mit dem Leibniz-Institut für Präventionsforschung und Epidemiologie in Bremen, institutionalisiert im Wissenschaftsschwerpunkt „Health Sciences Bremen“. Dort haben sich Schwerpunkte in Epidemiologie, Prävention und Versorgungsforschung herausgebildet;

- die Pettenkofer School of Public Health der LudwigMaximilians-Universität, München, kooperiert mit dem benachbarten Helmholtz-Zentrum für Gesundheit und Umwelt sowie dem Bayerischen Landesamt für Gesundheit und Lebensmittelsicherheit/Öffentlichen Gesundheitsdienst;

- das Institut für Public Health der Universität Heidelberg hat seinen Schwerpunkt im Bereich Global Health und kooperiert mit vielen Institutionen der internationalen Zusammenarbeit;

- die Hochschule Fulda kooperiert im Forschungsverbund für Sozialrecht und Sozialpolitik (FoSS) eng mit der Universität Kassel und dem Bundessozialgericht in Kassel und weist einen weiteren Schwerpunkt in der Qualifikation von Gesundheitsfachberufen in Public Health auf.

Für ein hochgradig interdisziplinäres Gebiet wie Public Health hat sich dieser Weg der Profilbildung einzelner Standorte bewährt, zumal er regional die unterschiedlich verteilten Stärken benachbarter Disziplinen berücksichtigen kann. Diese Art der Zusammenarbeit ist für das auch in der Stellungnahme vertretene Verständnis der Interdisziplinarität und der „Health in all Policies“ essenziell und darf nicht mit einer „Zersplitterung“ verwechselt werden. Auch die dort genannten Referenzländer verfolgen diesen Ansatz. Die in der Stellungnahme favorisierte Zentralisierung und vorgesehene Abwertung der Forschungsautonomie der Universitäten erhöht die Gefahr, den Wissenschaftsstandort Deutschland in diesem Bereich nachhaltig zu schwächen.

In der Lehre bieten alle Einrichtungen ein vergleichbares Kerncurriculum an. In den Wahlpflichtbereichen drückt sich das spezifische Profil der unterschiedlichen Standorte aus und sichert die Verknüpfung von Forschung und Lehre. Die im Papier geforderte Qualitätssicherung findet bereits seit vielen Jahren im Rahmen von Akkreditierungen nach dem Prinzip des peerreviews statt. Ein fachlicher und didaktischer Austausch erfolgt unter anderem über die Gremien der Deutschen Gesellschaft für Public Health (DGPH). Für ein interdisziplinäres Gebiet wie Public und Global Health ist, anders als bspw. im Medizinstudium, eine weitere Vereinheitlichung nicht zielführend. 
Eine zusätzliche Form der „Zersplitterung“ wird in der Trennung von universitärer Ausbildung und dem öffentlichen Gesundheitsdienst (ÖGD) gesehen. Tatsächlich arbeiten die universitären Public-Health-Ausbildungseinrichtungen und der Öffentliche Gesundheitsdienst sowohl auf Bundesebene (der Bundesverband der Ärztinnen und Ärzte des Öffentlichen Gesundheitsdienstes (BVÖGD) ist Mitglied in der DGPH), wie auch auf regionaler Ebene in Forschung, Lehre und Translation kontinuierlich zusammen. Richtig ist, dass, anders als für die sozialversicherungsfinanzierte Gesundheitsversorgung, für den steuerfinanzierten ÖGD kaum Forschungsmittel zur Verfügung stehen. Diese Lücke wird über die Kooperationen mit Hochschulen abgemildert, aber nicht geschlossen.

\section{Angebliche inhaltliche Versäumnisse von Public und Global Health in Deutschland}

Aus Sicht der Stellungnahme wurde wichtigen Themen und Bereichen bislang zu wenig Aufmerksamkeit gewidmet. Diese Behauptung beruht auf schlichter Unkenntnis der Aktivitäten: So heißt es in der Stellungnahme „Es sind strategische Beziehungen zu politischen Entscheidungsträgern und zur Zivilgesellschaft zu entwickeln, wie auch zu praxisorientierten Organisationen in Deutschland wie dem IQWIG (Institut für Qualität und Wirtschaftlichkeit im Gesundheitswesen) oder AQUA (Institut für angewandte Qualitätsförderung und Forschung im Gesundheitswesen), solche in der EU wie dem ECDC und weltweit, insbesondere zur WHO ... Die Bewertung von Gesundheitswirkungen (HTA) ist ein wichtiges Werkzeug, um die Folgen politischer Entscheidungen für andere Bereiche auszuloten“ (S. 67).

Fakt ist dagegen, dass

- die Zusammenarbeit zwischen akademischer Public Health und dem IQWiG kaum enger sein könnte (u.a. Bearbeitung von Aufträgen, gemeinsame Methodenentwicklung, Mitgliedschaften im Beirat, Vorträge in Studiengängen, Betreuung von Dissertationen);

- der Leiter des AQUA-Institutes in Personalunion eine universitäre Professur für Allgemeinmedizin und Versorgungsforschung innehat und der Austausch mit der akademischen Public Health entsprechend eng ist;

- die deutsche Public-Health-Community sehr erfolgreich eine Vielzahl von Projekten für die $E U$ und das ECDC durchführt;

- mehrere universitäre Institute aus dem Public-Health-Bereich in Deutschland als offizielle WHO collaborating centres arbeiten und viele Mitglieder der Community ihre Expertise in die Arbeit der WHO und ihrer Regionalorganisationen einbringen;

- HTA in Deutschland vor mehr als 15 Jahren aus einer universitären Netzwerkförderung durch das Bundesministerium für Gesundheit hervorgegangen ist, bevor es institutionell (DIMDI, IQWiG) etabliert wurde. Von den 5 aktuell laufenden EU-Projekten zu HTA finden 4 mit deutscher Beteiligung statt, eines davon wird von einer deutschen Universität koordiniert. Vor diesem Hintergrund wirkt die Aufforderung, Public Health möge sich doch mit den o.g. Bereichen beschäftigen, für alle Personen, die mit der Materie vertraut sind, mehr als befremdlich. Analog zu den o.g. Themen wird auch Public Health Genomics als ein Thema genannt, das von der Public-Health-Community übersehen worden wäre. Tatsächlich steht Public Health Genomics aktuell weder in Deutschland, noch in den meisten anderen Ländern im Zentrum der Aktivitäten von Public und Global Health. Das liegt allerdings nicht daran, dass das Thema in Deutschland nicht wahrgenommen worden wäre. Bereits im Jahr 2006 wurde das Deutsche Zentrum für Public Health Geno- mics an der Hochschule Bielefeld gegründet. Das (damalige) Landesinstitut für den Öffentlichen Gesundheitsdienst NRW koordinierte von 2007-2009 das erste EU-finanzierte Netzwerk „Public Health Genomics European Network (PHGEN I)“. Leider haben sich viele Versprechen von Public Health Genomics bisher nicht einlösen lassen. Es scheint daher nicht zielführend, aus dem ohnehin knappen Budget für Public Health weitere Mittel für das Gebiet der „-omics“ zu entnehmen, das seit vielen Jahren über eigene öffentliche Förderschienen und durch die Industrie in Größenordnungen alimentiert wird, die durch die Finanzierung der Public- und Global-Health-Forschung nicht annähernd erreicht werden [2].

\section{Leistungsfähigkeit von Public und Global Health in Deutschland}

An mehreren Stellen wird in der Stellungnahme betont, dass Public und Global Health in Deutschland weniger leistungsfähig sei, als in vergleichbaren Ländern. Diese Erkenntnis ist nicht neu und wird seit vielen Jahren von verschiedenen Akteuren, unter anderem der DGPH, konstatiert [3].

In der Stellungnahme der Akademien wird eine bibliometrische Analyse herangezogen, die zeigt, dass Deutschland im internationalen Ländervergleich - gemessen an der Zahl der Publikationen in definierten Zeitschriften - an neunter Stelle liegt. Allerdings sagt dieser Wert für sich genommen wenig aus: So wurden für die Vergleichsländer die führenden nationalen Public-Health-Zeitschriften in der jeweiligen Landessprache einbezogen, während diese für Deutschland nicht berücksichtigt wurden („Das Gesundheitswesen“ und „Bundesgesundheitsblatt-Gesundheitsforschung-Gesundheitsschutz“). Gleichzeitig bilden die Zeitschriften in der Stichprobe nur einen Bruchteil der möglichen Publikationsorgane ab. Dezidiert interdisziplinäre Arbeiten, die in Fachzeitschriften außerhalb einer engeren Public-Health-Zuordnung veröffentlicht werden, bleiben unberücksichtigt [4].

Allerdings lassen sich die Zahlen nutzen, um Trends zu analysieren: die Zahl der jährlichen Publikationen hat sich in den untersuchten Zeitschriften während des Beobachtungszeitraums (2000-2012) in Deutschland vervierfacht, während sie sich auf globaler Ebene lediglich verdoppelt hat. Leider findet sich ausgerechnet diese Aussage nicht in der Stellungnahme, sondern muss in der Anlage recherchiert werden [4]. Wenn sich also eine valide Aussage aus der bibliometrischen Analyse ableiten lässt, dann ist es die, dass die Forschung in Deutschland sich für den gewählten Indikator in den letzten 12 Jahren im globalen Vergleich weit überproportional entwickelt hat. Wie die kategorische Forderung nach weitreichenden strukturellen Reformen mit diesem Trend zusammenpassen soll, ist nicht nachvollziehbar.

\section{Fazit und Ausblick}

$\nabla$

Public und Global Health haben in Deutschland unbestritten schlechtere strukturelle Voraussetzungen als in den meisten vergleichbaren Ländern. Daran etwas zu verändern ist ein wichtiges Ziel. Es ist daher grundsätzlich zu begrüßen, dass sich die wissenschaftlichen Akademien den Forderungen einer materiellen und immateriellen Aufwertung von Public und Global Health in Deutschland anschließen.

Die Stellungnahme enthält eine Reihe von richtigen und wertvollen Vorschlägen, die aber allgemein gehalten und überwie- 
gend bereits bekannt sind. Dagegen laufen einige der konkret formulierten Empfehlungen Gefahr, die positiven Entwicklungen der letzten Jahre umzukehren. Diese Empfehlungen, welche die Rolle der Universitäten vornehmlich auf die Lehre reduzieren möchten und eine Umschichtung zugunsten zentraler Strukturen vorsehen, werden durch keine empirischen oder theoretischen Analysen gestützt. Stattdessen knüpfen sie an das Dokument der Helmholtz - Gemeinschaft „Helmholtz 2020 Zukunftsgestaltung durch Partnerschaft“ aus dem Jahr 2012 an, in dem den Universitäten eine gegenüber der HelmholtzGemeinschaft nachgeordnete Rolle zugedacht wird und das entsprechend von vielen Seiten kritisiert wurde [5].

Der inhaltliche Fokus der Stellungnahme, eine Stärkung von Public Health Genomics im Rahmen von Public Health, wird in der nationalen und internationalen Public-Health-Community als wichtiges, aber nicht prioritäres Thema für Public und Global Health wahrgenommen.

Für die Federführung der nächsten Schritte schlagen sich die beteiligten Akademien selbst vor, da sie als „unabhängige Einrichtungen ...eine geeignete Plattform [seien], um sämtliche Akteure zusammenzubringen“ (S. 72). Die Zusammenstellung der Autorinnen und Autoren der Stellungnahme, bei der Expertinnen und Experten von Public Health aus Deutschland mit wenigen Ausnahmen nicht berücksichtigt wurden, lässt nachhaltige Zweifel an dieser Fähigkeit aufkommen.

\section{Um Public und Global Health in Deutschland wirklich} zu stärken müssen

- die vorhandenen Public-Health-Standorte mit ihren individuellen Stärken und Profilen unterstützt und ihr Ausbau gefördert werden,

- Public-Health-Themen systematisch in nationale Förderprogramme zur Gesundheitsforschung integriert werden

- die Forschungsförderung und Förderpraxis an interdisziplinären Public-Health-Themen ausgerichtet werden.
Interessenkonflikt: Die Autoren und Autorinnen repräsentieren den Vorstand der Deutschen Gesellschaft für Public Health (DGPH). Die DGPH ist die wissenschaftliche Dachgesellschaft von Public Health und vertritt bundesweit 47 Mitgliedsorganisationen aus Fachgesellschaften, Hochschulen und anderen Institutionen, die sich mit Public Health befassen.

AG war zu einem Workshop der wissenschaftlichen Akademien eingeladen. Er hatte keinen Einfluss auf den Wortlaut der Stellungnahme.

\section{Institute}

${ }^{1}$ Abteilung Versorgungsforschung, Institut für Public Health und

Pflegeforschung, Universität Bremen

${ }^{2}$ Health Sciences Bremen

${ }^{3}$ Public Health Institute Fulda, Hochschule Fulda

${ }^{4}$ Abteilung Sozialepidemiologie, Institut für Public Health und

Pflegeforschung, Universität Bremen

${ }^{5}$ Institut für Epidemiologie, Sozialmedizin und Gesundheitssystemforschung,

Medizinische Hochschule Hannover

${ }^{6}$ Fakultät für Gesundheitswissenschaften/AG Epidemiologie \& International

Public Health, Universität Bielefeld

${ }^{7}$ Department of Community Health, Hochschule für Gesundheit Bochum

${ }^{8}$ Arbeitsbereich Public Health: Prävention und psychosoziale

Gesundheitsforschung, Freie Universität Berlin

\section{Literatur}

1 Nationale Akademie der Wissenschaften Leopoldina, acatech - Deutsche Akademie der Technikwissenschaften und Union der deutschen Akademien der Wissenschaften. Public Health in Deutschland - Strukturen, Entwicklungen und globale Herausforderungen. Halle (Saale), 2015

2 Joyner MJ, Paneth $N$. Seven Questions for Personalized Medicine. JAMA; 2015. doi: 10.1001/jama.2015.7725. [Epub ahead of print].

3 Gerlinger T, Babitsch B, Blättner B et al., Deutsche Gesellschaft für Public Health. Situation und Perspektiven von Public Health in Deutschland Forschung und Lehre. Positionspapier der Deutschen Gesellschaft für Public Health e. V. Gesundheitswesen 2012; 762-766

4 Donner P, Chi PS, Aman V. Bibliometric study for German National Academy of Sciences Leopoldina in the disciplines Public Health and Epidemiology. Institut für Forschungsinformation und Qualitätssicherung; Berlin: 2014

5 Helmholtz-Gemeinschaft. Helmholtz 2020 - Zukunftsgestaltung durch Partnerschaft. Der Beitrag der Helmholtz-Gemeinschaft zur Weiterentwicklung des Wissenschaftsstandorts Deutschland. ohne Ort: Helmholtz-Gemeinschaft; 2012 